\title{
Uma Solução de Decisão de Handover inter-V-Cell para Veículos Conectados em Redes 5G Ultradensas
}

\author{
Carlos R. Storck ${ }^{1,3}$, Efrem E. de O. Lousada ${ }^{2,3}$, Guilherme G. de O. Silva ${ }^{3}$, \\ Fátima Duarte-Figueiredo ${ }^{3}$, Raquel A. F. Mini $^{3}$
}

${ }^{1}$ Centro Federal de Educação Tecnológica de Minas Gerais (CEFET-MG)

${ }^{2}$ Instituto Federal de Minas Gerais (IFMG)

${ }^{3}$ Pontifícia Universidade Católica de Minas Gerais (PUC Minas)

storck@cefetmg.br, efrem.lousada@ifmg.edu.br, guigalv@hotmail.com,

ffatimafig, raquelmini\}epucminas.br

\begin{abstract}
The fifth generation (5G) cellular network requires new solutions to new network requirements, for example, support for high speed mobility. This paper presents a handover decision solution for connected vehicles to ultradense $5 G$ networks. Its main goal is to treat high vehicular mobility and provide performance gains in handover for vehicles, since the densification of the cellular network causes, despite the expected capacity gain, difficulties in the cells selection, a greater number of failed and unnecessary handovers (pingpong effect), longer delays and energy consumption, and high packet losses. The solution is based on virtual cells (V-Cells). The selection of the best cells is made based on complex network metrics to compose a virtual cell, as well as other criteria such as signal strength, distance and speed. The solution relies on the software-defined network (SDN) controller which has centralized network information. Simulations were conducted in Network Simulator, ns-3. The results show that the solution is effective in the treatment of vehicle handover.
\end{abstract}

Resumo. A rede de celular de quinta geração $(5 G)$ requer novas soluções para novos requisitos de rede, como o suporte à mobilidade para altas velocidades. Este trabalho apresenta uma solução de decisão de handover para veículos conectados em redes $5 G$ ultradensas. Ele tem como objetivo principal tratar a alta mobilidade veicular e proporcionar ganhos de desempenho em handover para veículos, uma vez que a densificação da rede celular causa, apesar do ganho de capacidade esperado, dificuldades na seleção de células, maior número de handovers falhos e desnecessários (efeito ping-pong), maiores atrasos e consumo de energia, e altas perdas de pacotes. A solução tem como base a formação de células virtuais (V-Cells). A seleção de melhores células é feita com o auxílio de métricas de redes complexas para compor uma célula virtual, assim como critérios como potência de sinal, distância e velocidade. A solução se apoia em um controlador de rede definida por software (SDN) para tomada de decisão, que possui informação centralizada da rede. Simulações foram conduzidas no simulador de rede, ns-3. Os resultados obtidos mostram que a solução é eficaz no tratamento de handover para veículos. 


\section{Introdução}

Em todo o mundo, sistemas de condução para assistência aos motoristas têm sido desenvolvidos utilizando comunicação de redes veiculares (VANETs - Veicular Ad Hoc Networks). As redes de comunicação sem fio, em especial as redes de celulares, são os pilares de infraestrutura para apoiar tais sistemas. Os sistemas voltados para veículos possuem novos requisitos de rede, tais como alta taxa de transmissão (entre 1 e $20 \mathrm{Gbps}$ ), densidade massiva de conexões (estimativa em 1 milhão de dispositivos e veículos conectados por $\mathrm{km}^{2}$ ), comunicações ultra confiáveis de baixa latência (1 ms) e suporte à mobilidade para altas velocidades (até $500 \mathrm{~km} / \mathrm{h}$ ) [ITU 2015], [Minoli and Occhiogrosso 2019]. Estes requisitos visam garantir o máximo conforto e tornar a condução do veículo a mais segura possível.

As aplicações para veículos envolvem a comunicação entre veículos (V2V Vehicle-to-Vehicle), entre veículos e infraestrutura (V2I - Vehicle-to-Infrastructure) ou entre veículos e outros elementos/tudo (V2X - Vehicle-to-Everything). Técnicas eficientes de handover (HO) para usuários em altas velocidades são necessárias para o sucesso das comunicações entre veículos e tudo. Em razão da mobilidade dinâmica associada às altas velocidades, veículos apresentam handovers e desconexões frequentes, além da densificação da rede causar, apesar do ganho de capacidade esperado: dificuldades na seleção de células, um maior número de handovers falhos e desnecessários (conhecidos como efeito ping-pong), maiores atrasos e consumo de energia, altas perdas de pacotes e uma má qualidade de experiência [Tayyab et al. 2019], o que torna o processo de handover mais complicado, sendo um desafio realizá-lo de maneira eficaz.

O objetivo deste artigo é apresentar uma solução de handover para veículos conectados em redes 5G ultradensas (UDN - Ultradense Network). Com uma visão unificada da rede, proporcionada pela adoção de controlador SDN, a solução passa a ter ciência do contexto geral da rede, centralizando algumas decisões. O paradigma SDN surgiu há alguns anos e permite que uma rede seja diretamente programável, dissociando o plano de controle do plano de dados. O procedimento adotado é híbrido, centrado: no usuário para a escolha de melhores células para formar uma célula virtual, e na rede para a execução propriamente dita do handover. Célula virtual é uma abstração de um conjunto de recursos de células físicas vistas como uma única macro-célula, gerenciada logicamente. Para isso, a virtualização de funções de rede (NFV - Network Functions Virtualization) dissocia funções de rede da infraestrutura física. Ambas as tecnologias, SDN e NFV, permitem simplificar a implantação e o gerenciamento de serviços em redes ultradensas, tais como 5G [Gharsallah et al. 2018]. Desta forma, as células virtuais são atualizadas dinamicamente, o que proporciona handovers mais rápidos e eficazes. A solução seleciona células por critérios de qualidade (especificados pelo 3GPP - 3rd Generation Partnership Project) e por métricas de redes complexas [Lousada et al. 2019], na formação de células virtuais. Os multicritérios utilizados diferenciam este trabalho das soluções propostas na literatura e proporcionam ganhos de desempenho em handover: diminuição no tempo de execução do $\mathrm{HO}$, diminuição no número de HOs desnecessários e falhos, e diminuição no número de pacotes perdidos.

Este trabalho está organizado da seguinte forma: a Seção 2 apresenta os trabalhos relacionados; a Seção 3 descreve a solução proposta; a Seção 4 apresenta a metodologia adotada; a Seção 5 avalia os resultados da simulação; e a Seção 6 discorre as conclusões. 


\section{Trabalhos Relacionados}

Ao longo dos anos, diversos trabalhos foram desenvolvidos para tratamento de handover. No entanto, novas propostas são necessárias para as comunicações veiculares em redes 5G ultradensas. A Tabela 1 apresenta as abordagens adotadas nos trabalhos relacionados.

Tabela 1. Abordagens adotadas nos trabalhos relacionados

\begin{tabular}{|c|c|c|c|}
\hline Referência & $\begin{array}{l}\text { Principais } \\
\text { Características }\end{array}$ & Destaques & Ponderações \\
\hline [Polese et al. 2017] & $\begin{array}{l}\text { Protocolo de conectividade } \\
\text { dupla. }\end{array}$ & $\begin{array}{l}\text { Protocolo implementado no } \\
\text { simulador ns-3. }\end{array}$ & $\begin{array}{l}\text { Simula o cenário urbano em uma } \\
\text { área de } 200 \times 115 \text { metros. }\end{array}$ \\
\hline [Mouawad et al. 2018] & $\begin{array}{l}\text { Handover centrado na rede } \\
\text { é de responsabilidade do } \\
\text { controlador SDN. }\end{array}$ & $\begin{array}{l}\text { Seleção da rede ocorre com } \\
\text { base na teoria da utilidade. }\end{array}$ & $\begin{array}{l}\text { Simulações consideram } 802.11 \mathrm{p} \\
\text { e LTE para veículos (LTE-V) } \\
\text { através do Mininet-WiFi. }\end{array}$ \\
\hline [Rizvi and Akram 2018] & $\begin{array}{l}\text { Gerenciamento de handover } \\
\text { V2X baseado em } 5 \mathrm{G} \text { SDN; se } \\
\text { realiza através da interface X } 2 \text {. }\end{array}$ & $\begin{array}{l}\text { Handover é o principal evento } \\
\text { acionado em técnicas de corte } \\
\text { de rede e virtualização. }\end{array}$ & $\begin{array}{l}\text { Simulações considerando apenas } \\
\text { parâmetros de rede LTE. }\end{array}$ \\
\hline [Gharsallah et al. 2018] & $\begin{array}{l}\text { Solução de handover com } 4 \\
\text { fases: coleta, processamento, } \\
\text { criação de células virtuais e } \\
\text { execução de handover. }\end{array}$ & $\begin{array}{l}\text { As células virtuais consideram } \\
\text { o tamanho das células de } \\
\text { acordo com a mobilidade. } \\
\text { Validação por MATLAB. }\end{array}$ & $\begin{array}{l}\text { As velocidades não são } \\
\text { explicitadas. Processo de } \\
\text { HO tradicional LTE (Long Term } \\
\text { Evolution). }\end{array}$ \\
\hline [Peng et al. 2019] & $\begin{array}{l}\text { Estratégias de handover para } \\
\text { VANETs (camada de rede). }\end{array}$ & $\begin{array}{l}\text { Handover vertical é centrado } \\
\text { no usuário ou na rede. }\end{array}$ & $\begin{array}{l}\text { É fornecida apenas uma visão das } \\
\text { estratégias. }\end{array}$ \\
\hline [Zang et al. 2019] & $\begin{array}{l}\text { Gerenciamento de } \\
\text { verticais em } \begin{array}{r}\text { havers } \\
\text { heterogêneas. }\end{array}\end{array}$ & $\begin{array}{l}\text { Proposta baseada em } \\
\text { MDP usando velocidade e } \\
\text { localização do usuário. }\end{array}$ & $\begin{array}{l}\text { A velocidade em cenários de } \\
\text { alta mobilidade foi de } 4,57 \mathrm{~m} / \mathrm{s} \\
(16,46 \mathrm{~km} / \mathrm{h}) \text {. }\end{array}$ \\
\hline [Oliveira et al. 2019] & $\begin{array}{l}\text { É apresentado o SoftH, um } \\
\text { mecanismo de handover suave. }\end{array}$ & Proposta baseada em SDN. & $\begin{array}{l}\text { Considera conexões ativas em até } \\
\text { duas células potenciais apenas, e } \\
\text { foi avaliada em } 4 \mathrm{G} \text { LTE. }\end{array}$ \\
\hline
\end{tabular}

As redes 5G são ultradensas e heterogêneas, fato que, por si só, gera handovers frequentes, indesejados, desnecessários (efeito ping-pong) e falhos, além de aumentar o tempo do processo de handover [Bilen et al. 2017]. Diferentes estratégias de handover são apresentadas em outros trabalhos [Polese et al. 2017, Mouawad et al. 2018, Rizvi and Akram 2018, Gharsallah et al. 2018, Peng et al. 2019, Zang et al. 2019]. Um mecanismo de handover suave (SoftH), que utiliza o conceito de SDN é capaz de realizar decisões de handovers de uma maneira mais assertiva [Oliveira et al. 2019]. Este trabalho apresenta uma evolução do mecanismo SoftH, que é portanto um novo mecanismo de handover com foco em comunicações veiculares 5G. Mas outras estratégias de decisão, distintas das que foram empregadas neste trabalho, são encontradas na literatura para tratar e reduzir o número de handovers [Souza et al. 2020], tais como lógica fuzzy, processo hierárquico analítico (AHP - Analytical Hierarchical Process), Q-learning, TOPSIS (Technique for Order Preference by Similarity to Ideal Solution) e aprendizado por reforço.

\section{Abordagem Proposta}

Conforme definido por padrões estabelecidos pelo 3GPP, um handover pode ser desencadeado por diferentes eventos. Na maior parte das vezes, há limiares de potência mínima de sinal, que, quando ultrapassados, disparam handovers. A maioria das propostas usa o evento $\mathrm{A} 2$ ou o evento $\mathrm{A} 3$, ambos usados neste trabalho. O procedimento é mostrado na Figura 1, que representa o ciclo de funcionamento da operação de handover executado: cada veículo é vinculado a um conjunto de antenas, nas estações base (BSs - Base Stations), que compõe virtualmente uma $V$-Cell, que por sua vez é criada e 
gerenciada por NFV. A virtualização é necessária à instanciação de células virtuais e recebe os parâmetros do controlador. O controlador SDN é necessário para o prévio conhecimento da topologia, da realização de cálculos e da tomada de decisão.

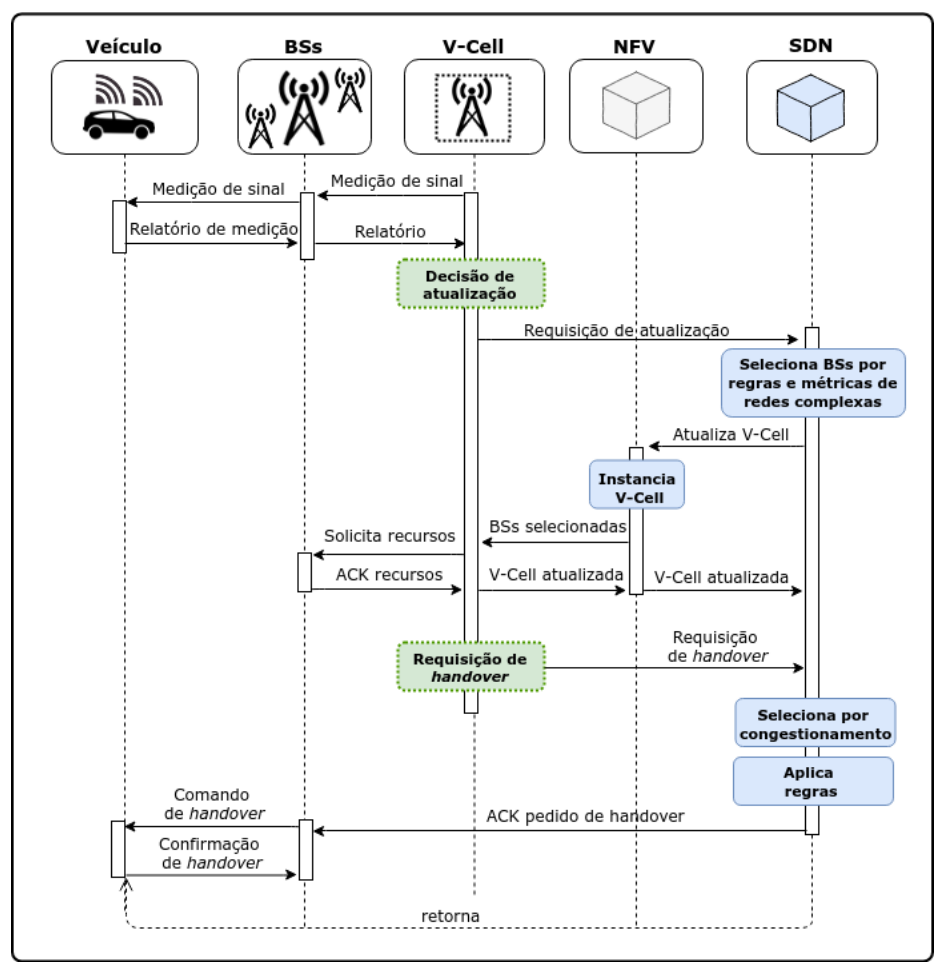

Figura 1. Procedimento de handover da solução desenvolvida.

Para minimizar os problemas decorrentes de alta velocidade veicular, há a seleção das melhores células, formando uma célula virtual para os veículos. A Figura 2 ilustra a aplicação das instâncias de células virtuais de acordo com a velocidade dos veículos em uma topologia 5G UDN, considerando os seguintes tamanhos para o melhor dos casos: células pequenas (picocells) para velocidades entre zero e $30 \mathrm{~km} / \mathrm{h}$; células médias (microcells) para velocidades entre 31 e $120 \mathrm{~km} / \mathrm{h}$; e células grandes (macrocells) para velocidades extremamente altas (entre 121 e $500 \mathrm{~km} / \mathrm{h}$ ). As subseções a seguir apresentam mais detalhes sobre as operações adotadas pela solução neste trabalho.

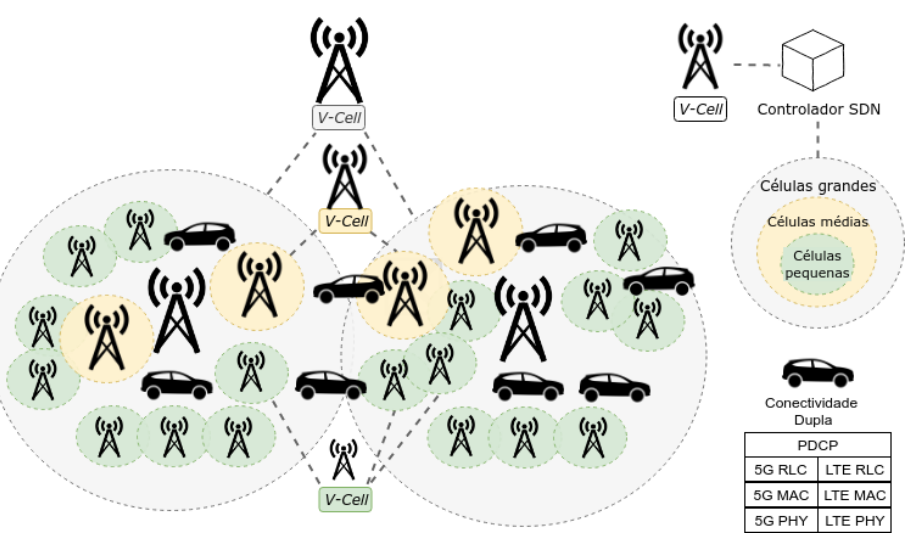

Figura 2. Conexões veiculares em $5 G$ e LTE por V-Cells. 


\subsection{Células Virtuais}

A adoção de $V$-Cells permite que abordagens sejam criadas centradas no usuário. Uma vez verificadas as células disponíveis, de acordo com as regras apresentadas na Tabela 2, a fase de seleção de antenas candidatas para atualização das $V$-Cells é realizada. Conforme apresentado na Tabela 2, os limiares de velocidade foram definidos baseados no 3GPP TR 38.913 e que foram adequados por tipo de célula (pequenas, médias e grandes). Neste trabalho assumimos células pequenas com um raio de cobertura entre 100 a 200 metros (m), células médias entre 200 a 500 m, e células grandes a partir de $500 \mathrm{~m}$.

Tabela 2. Regras de V-Cells por faixas de velocidade e tamanho das células

\begin{tabular}{llll}
\hline & Baixa velocidade & Alta velocidade & Velocidade muito alta \\
$\mathbf{V}$-Cell & $\mathbf{0 - 3 0} \mathbf{~ k m} / \mathbf{h}$ & $\mathbf{3 1 - 1 2 0} \mathbf{~ k m} / \mathbf{h}$ & $\mathbf{1 2 1 - 5 0 0 ~} \mathbf{~ k m} / \mathbf{h}$ \\
\hline Opção 1 & Células pequenas & Células médias & Células grandes \\
Opção 2 & Células médias & Células grandes & Células médias \\
Opção 3 & Células grandes & Células pequenas & Células pequenas \\
\hline
\end{tabular}

A escolha dos componentes para calcular a probabilidade de seleção, baseouse na posição do veículo, no grau e na centralidade da intermediação de uma BS na rede. Todas as possíveis células (previamente conhecidas pelo controlador SDN) são analisadas, selecionando até três potenciais células candidatas $(k=3)$, se disponíveis na topologia. A probabilidade de seleção da célula candidata, denominada de " $p S$ ", é calculada pela razão entre o produto da probabilidade associada ao grau das estações base ( $p B S d e g r)$ e a probabilidade associada ao betweenness ( $p B e t w$ ) dividida pela distância (em metros) do veículo à $\mathrm{BS}$ (distVehBS), formulada pela Equação (1):

$$
p S=\frac{p B S \operatorname{degr} \times p B e t w}{\operatorname{distVehBS}}
$$

Para a seleção de células candidatas, é interessante a escolha das BSs que possuam menor valor de grau. Ter o menor grau não indica necessariamente que uma célula tem capacidade de transmissão, pois depende das aplicações que estão em execução. Desta forma, um controle de congestionamento e balanceamento também será empregado, e realizado por uma função de teoria da utilidade, empregada também em outro trabalho

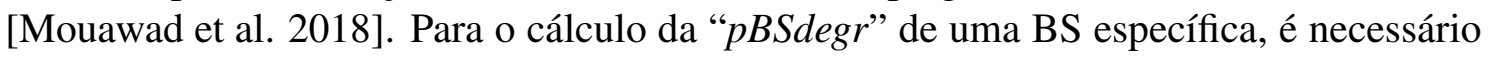
que as BSs conheça o grau do vértice de todas as BSs vizinhas e veículos conectados. O cálculo de " $p B S$ degr" é dado pela diferença entre um e a razão do grau de vértice ( $v D e g)$, obtido pela contagem do número de veículos e BSs alcançáveis (essas informações são mantidas na tabela de vizinhos da própria BS), pela soma dos graus de vértice de seus vizinhos (VDegNeig), conforme mostrado na Equação (2):

$$
p B S \operatorname{deg} r=1-\frac{v D e g}{V D e g N e i g}
$$

Seguindo o mesmo raciocínio da métrica anterior, é interessante a escolha de BSs que possuam um menor valor de betweenness. O cálculo da "pBetw" normalizada, para medir a importância da BS, é mostrado na Equação (3), onde " $g_{s t}$ " é o caminho mais curto da fonte ( $s$ - source) para destino $(t)$, e " $n_{s t}^{i}$ " é o caminho mais curto de " $s$ " para " $t$ " passando pelo vértice " $i$ ", considerando " $N$ " como o número de vértices:

$$
p B e t w=\frac{1}{\frac{(N-1) \times(N-2)}{2}} \sum_{s \neq i \neq t} \frac{n_{s t}^{i}}{g_{s t}}
$$




\subsection{Regras de Velocidade Média e Qualidade do Serviço}

Uma vez instanciada a nova célula virtual, as atuais BSs, rotuladas como célula virtual atual ( cvc - current virtual cell) durante a atualização, são inspecionadas, imediatamente e em conjunto, para execução do handover entre células virtuais distintas (inter-V-Cell), comparando com as novas células selecionadas e rotuladas como célula virtual vizinha ( nvc - neighboring virtual cell). Nesse processo, é importante observar outros fatores relevantes durante o processo de decisão de handover. Um deles é a velocidade média de movimento do veículo. A primeira classificação realizada é em relação à velocidade, separando os veículos em grupos distintos, para que um veículo seja classificado de acordo com a qualidade do serviço (QoS - Quality of Service) e a sua respectiva identificação de classe, o seu QCI (QoS Class Identifier) ou com o seu 5QI (QCI para $5 \mathrm{G})$, que define a qualidade da transmissão. Os veículos são divididos em grupos por prioridade. Nesta proposta, valores de QCI e 5QI são adicionados conforme a especificação TS 23.501, do 3GPP. As regras de handover foram definidas para cada grupo, definidas como intervalos de RSRQ, que é o indicador de qualidade do sinal e pela força do sinal de referência recebido (RSRP - Reference Signals Received Power). Os intervalos são usados para decisões de limite de handover de uma $c v c$ para uma $n v c$. Essas regras são apresentadas na Tabela 3.

Tabela 3. Regras de handover por faixas de velocidade em 5G UDN

\begin{tabular}{|c|c|c|c|}
\hline QCI (4G) & $\begin{array}{l}\text { Baixa velocidade } \\
0-30 \mathrm{~km} / \mathrm{h}\end{array}$ & $\begin{array}{l}\text { Alta velocidade } \\
31-120 \mathrm{~km} / \mathrm{h}\end{array}$ & $\begin{array}{l}\text { Velocidade muito alta } \\
121-500 \mathrm{~km} / \mathrm{h}\end{array}$ \\
\hline $1,2,3$ and $4(\mathrm{GBR})$ & RSRQ $c v c<$ RSRQ $n v c$ & RSRQ $c v c<$ RSRQ $n v c$ & RSRQ $c v c<$ RSRQ $n v c$ \\
\hline 5 and 7 (non-GBR) & RSRQ $c v c<=23(-8.5 \mathrm{~dB})$ & RSRQ $c v c<=24(-8.0 \mathrm{~dB})$ & RSRQ $c v c<=25(-7.5 \mathrm{~dB})$ \\
\hline $6,8,9$ (non-GBR) and default & RSRQ $c v c<=22(-9.0 \mathrm{~dB})$ & RSRQ $c v c<=23(-8.5 \mathrm{~dB})$ & RSRQ $c v c<=24(-8.0 \mathrm{~dB})$ \\
\hline 5QI (5G) & $\begin{array}{l}\text { Baixa velocidade } \\
0-30 \mathrm{~km} / \mathrm{h}\end{array}$ & $\begin{array}{l}\text { Alta velocidade } \\
31-120 \mathrm{~km} / \mathrm{h}\end{array}$ & $\begin{array}{l}\text { Velocidade muito alta } \\
121-500 \mathrm{~km} / \mathrm{h}\end{array}$ \\
\hline $65,67,75$ and $84(\mathrm{GBR})$ & RSRP $c v c<$ RSRP $n v c$ & RSRP $c v c<\operatorname{RSRP} n v c$ & RSRP $c v c<\operatorname{RSRP} n v c$ \\
\hline 69 and 79 (non-GBR) & $\operatorname{RSRP} c v c<50(-90 \mathrm{dBm})$ & $\operatorname{RSRP} c v c<60(-80 \mathrm{dBm})$ & $\operatorname{RSRP} c v c<70(-70 \mathrm{dBm})$ \\
\hline 70 (non-GBR) and default & $\operatorname{RSRP} c v c<40(-100 \mathrm{dBm})$ & $\operatorname{RSRP} c v c<50(-90 \mathrm{dBm})$ & $\operatorname{RSRP} c v c<60(-80 \mathrm{dBm})$ \\
\hline
\end{tabular}

Veículos podem viajar em alta velocidade, ou não. Quando não viajam em alta velocidade, tendem a não alterar sua medição do RSRQ ou RSRP repentinamente. Desta forma, eles podem permanecer conectados em suas células até atingirem níveis mínimos, que ainda representam conexões de boa qualidade. Quando viajam em alta velocidade, tendem a alterar seu RSRQ ou RSRP repentinamente, causando falhas no handover. Sendo assim, é importante definir valores de limite com um nível de segurança mais alto do que em cenários de baixa velocidade.

\section{Metodologia de Avaliação}

A abordagem proposta foi avaliada através do simulador de eventos discretos em rede (ns-3). Neste trabalho, foi utilizado o módulo ns3-mmWave (ns3 mmWave Cellular Network Simulator), que permite a adoção de interfaces de comunicação 5G e LTE simultaneamente. Também foi utilizado o módulo OFSwitch13 (OpenFlow 1.3 module for $n s-3)$, que permite a inclusão de um controlador SDN nos cenários, que neste estudo foi utilizado o próprio controlador OpenFlow 1.3. O modelo de mobilidade veicular foi criado através da simulação de mobilidade urbana (SUMO - Simulation of Urban Mobility). As aplicações que foram consideradas nas avaliações foram geradas através do modelo de tráfego "envio em massa" (Bulk-Send) e são classificadas de acordo com 
o seu QCI ou 5QI. As simulações foram executadas em um ambiente computacional de alto desempenho, fornecido pelo cluster F37 do CEFET-MG. Os cenários foram avaliados com intervalo de confiança de $95 \%$ para dez ensaios de simulação com duração de 100 segundos, como apresentado na Tabela 4, com base na literatura [Zang et al. 2019] e cenários de vias urbanas e rodovias (Figura 3) conforme documento 3GPP R1-164679.

\begin{tabular}{lll} 
& \multicolumn{2}{c}{ Tabela 4. Cenários simulados } \\
\hline Parâmetros & $\begin{array}{l}\text { Cenário I } \\
\text { Via urbana }\end{array}$ & $\begin{array}{l}\text { Cenário II } \\
\text { Rodovia }\end{array}$ \\
\hline Estrutura & 9 quarteirões $(250 \mathrm{~m} \mathrm{x} 433 \mathrm{~m})$ com 8 pistas de & 6 pistas de $4 \mathrm{~m}$ cada, 3 em cada direção $(2.000$ \\
& $3,5 \mathrm{~m}$ cada, 2 pistas em sentido horário & metros $)$ \\
Velocidades & 15,30 e $60 \mathrm{~km} / \mathrm{h}$ & 70,140 e $300 \mathrm{~km} / \mathrm{h}$ \\
Posicionamento & Processo de Poisson & Processo de Poisson \\
Espaçamento médio & 2.5 segundos $(\mathrm{s})$ & $2.5 \mathrm{~s}$ \\
Densidade & $20,60,120$ e 240 veículos $/ \mathrm{km}^{2}$ & 5,10 e 20 veículos $/ \mathrm{km} /$ pista \\
Número de gNodeB's & $20($ cenário denso) & 5 (cenário esparso $)$ \\
Potência (gNodeB) & $30 \mathrm{dBm}$ & $30 \mathrm{dBm}$ \\
Número de eNodeB's & 1 & 2 \\
Potência (eNodeB) & $46 \mathrm{dBm}$ & $46 \mathrm{dBm}$ \\
\hline
\end{tabular}

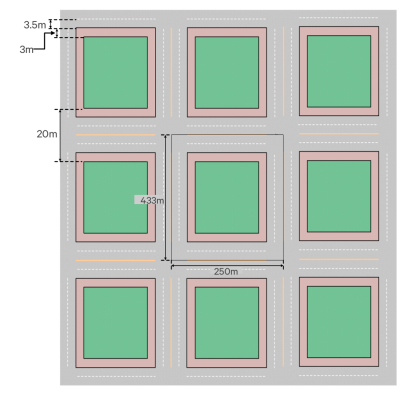

(a) Cenário I

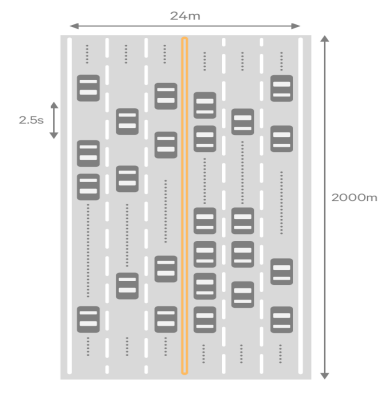

(b) Cenário II

Figura 3. Cenários de vias urbanas e rodovia (Adaptada de [Nguyen et al. 2017]).

O cenário I, apresentado na Figura 3(a), consiste em uma área urbana com nove quarteirões, cada uma com 250 x $433 \mathrm{~m}$ e 2 pistas em cada lado com 3,5 m cada, nas quais os veículos se movem em sentido horário para tornar possível as conversões em cada quadrante. Velocidades distintas foram adotadas (15, 30 e $60 \mathrm{~km} / \mathrm{h}$ ) para tornar possível a classificação das células e regras de handover por faixas de baixa velocidade e alta velocidade pela solução. As densidades de veículos para estradas urbanas foram definidas e classificadas como tráfego leve (20 veículos $\left./ \mathrm{km}^{2}\right)$, tráfego pesado (60 veículos $/ \mathrm{km}^{2}$ ), tráfego congestionado (120 veículos $\left./ \mathrm{km}^{2}\right)$ e tráfego altamente congestionado (240 veículos $/ \mathrm{km}^{2}$ ) [Silva et al. 2019].

O cenário II, apresentado na Figura 3(b), consiste em um rodovia com seis pistas e uma extensão de $2 \mathrm{~km}$, com veículos se movendo em direções opostas, ou seja, da parte superior para a inferior, nas três primeiras pistas, e da parte inferior para a superior, nas demais pistas. Velocidades distintas foram adotadas (70, 140 e $300 \mathrm{~km} / \mathrm{h}$ ) para tornar possível a classificação das células e regras de handover por faixas de alta velocidade e velocidade muito alta. As densidades consideradas para os cenários de rodovias foram de 5, 10 e 20 veículos/km/pista [Vukadinovic et al. 2018]. 


\section{Resultados e discussão}

As métricas avaliadas durante as simulações foram: taxa de perda de pacotes (PLR - Packet Loss Ratio), tempo médio de handovers (HO), taxa de handovers falhos e desnecessários. Pelos resultados obtidos com um intervalo de confiança de $95 \%$, o número médio de handovers por usuário foi de 7,8 e a PLR foi de 2,938\%. O tempo médio para execução do HO foi de $1,9590 \mathrm{~ms}$. Foram obtidas também excelentes taxas de HOs falhos e HOs desnecessários pela solução, de 2,884\% e 3,550\%, respectivamente. A Tabela 5 apresenta os resultados obtidos nas simulações dos dois cenários.

Tabela 5. Resultados obtidos

\begin{tabular}{|c|c|c|c|c|}
\hline Cenário I & PLR (\%) & Tempo médio de HO (ms) & HOs falhos (\%) & HOs desnecessários (\%) \\
\hline 20 veículos $/ \mathrm{km}^{2}$ & $2,687 \pm 0,377$ & $1,8665 \pm 0,2277$ & $2,777 \pm 1,071$ & $2,868 \pm 0,857$ \\
\hline 60 veículos $/ \mathrm{km}^{2}$ & $2,873 \pm 0,641$ & $1,9511 \pm 0,2090$ & $2,571 \pm 1,292$ & $3,375 \pm 0,701$ \\
\hline 120 veículos $/ \mathrm{km}^{2}$ & $3,207 \pm 0,623$ & $1,8440 \pm 0,2289$ & $3,428 \pm 1,589$ & $3,857 \pm 0,638$ \\
\hline 240 veículos $/ \mathrm{km}^{2}$ & $3,252 \pm 0,477$ & $2,1939 \pm 0,1532$ & $3,444 \pm 1,337$ & $3,791 \pm 0,153$ \\
\hline Cenário II & PLR (\%) & Tempo médio de HO (ms) & HOs falhos (\%) & HOs desnecessários (\%) \\
\hline 5 veículos $/ \mathrm{km} /$ pista & $2,722 \pm 0,416$ & $1,9833 \pm 0,2536$ & $2,175 \pm 1,289$ & $3,178 \pm 0,895$ \\
\hline 10 veículos $/ \mathrm{km} /$ pista & $2,786 \pm 0,433$ & $1,8235 \pm 0,4601$ & $2,475 \pm 1,587$ & $3,608 \pm 0,535$ \\
\hline \multirow[t]{2}{*}{20 veículos/km/pista } & $3,045 \pm 0,436$ & $2,0507 \pm 0,3022$ & $3,318 \pm 1,264$ & $4,174 \pm 0,405$ \\
\hline & $2,938 \%$ & $1,9590 \mathrm{~ms}$ & $2,884 \%$ & $\mathbf{3 , 5 5 0} \%$ \\
\hline
\end{tabular}

Para fins de validação, avaliamos e comparamos a proposta desenvolvida com os cenários apresentados pelos trabalhos da literatura: [Polese et al. 2017], [Rizvi and Akram 2018], [Prados-Garzon et al. 2016] e [Gharsallah et al. 2018]. Polese et al. [Polese et al. 2017] apresentou um número médio de eventos de $\mathrm{HO}$ entre $21 \mathrm{e}$ 43 e PLR entre $7 \%$ e 15\%, em um cenário de usuário único, considerando uma área de 200 x 115 m. Sob as mesmas condições, a solução desenvolvida apresentou um número médio de eventos de $\mathrm{HO}$ de 3,8 e PLR de 1,89\%. Considerando ainda os resultados gerais obtidos pelos cenários I e II, a solução apresenta uma redução significativa, entre $63 \%$ a $82 \%$, na quantidade de handovers executados por usuário, em razão do uso de células virtuais compostas pelas três melhores células centradas no usuário, além da baixa taxa de PLR, o que representa um considerável ganho na entrega de pacotes com sucesso.

Comparando os resultados sob as mesmas condições do trabalho de Rizvi e Akram [Rizvi and Akram 2018], em que o tempo de handover é de 13,24 ms em um cenário de usuário único, a solução apresenta uma média geral de tempo total de execução de handover de 1,4083 ms. Ao considerar a média geral de tempo total de execução de handover de 1,9590 ms obtida nos cenários I e II, houve um ganho equivalente a 85,20\%.

Comparando, ainda, com as análises de Prados-Garzon et al. [Prados-Garzon et al. 2016], em que o tempo de execução foi de 15,25 ms, em um cenário com área de 500 x $500 \mathrm{~m}$, contendo 20 BSs e 100 usuários se movendo a 21,6 $\mathrm{km} / \mathrm{h}(6 \mathrm{~m} / \mathrm{s})$, a solução apresenta um tempo total de execução de handover de 1,8233 ms. Ao considerar o resultado geral obtido de $1,9638 \mathrm{~ms}$ no ambiente de vias urbanas, ou seja, no cenário I, houve uma otimização equivalente a 87,12\%.

Em relação à sobrecarga e ao tempo de processamento, os resultados mostraram que o impacto do controlador SDN na solução foi irrelevante, alcançando bons resultados em relação à vazão e ao tempo médio de execução do handover, assim como observado no mecanismo SoftH [Oliveira et al. 2019]. Geralmente, a sobrecarga de sinalização tem um impacto significativo na taxa de vazão, o que não ocorreu. A Figura 4 mostra a 
função de distribuição cumulativa (CDF - Cumulative Distribution Function) da vazão (throughput) obtida para as velocidades adotadas com trânsito altamente congestionado (240 veículos $/ \mathrm{km}^{2}$ ) no cenário I e para 20 veículos $/ \mathrm{km} /$ pista no cenário II.

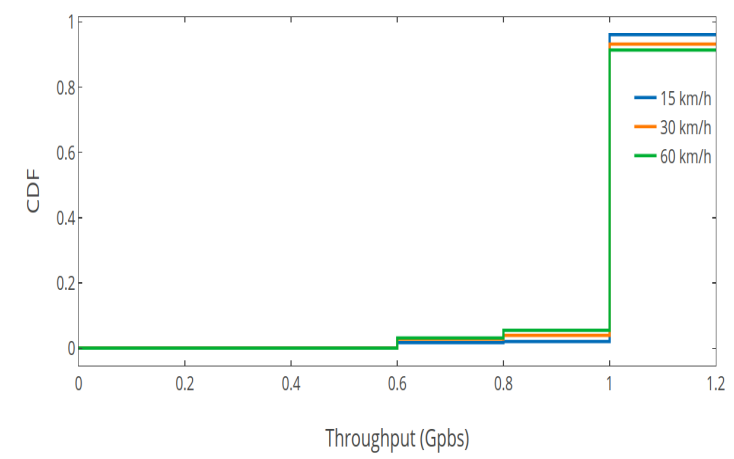

(a) Cenário I (240 veículos $/ \mathrm{km}^{2}$ )

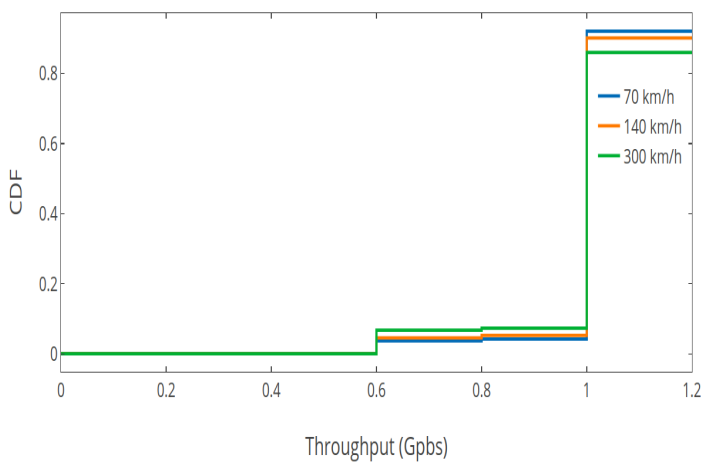

(b) Cenário II (20 veículos/ $\mathrm{km} /$ pista)

Figura 4. CDF do throughput obtido.

Na Figura 4(a), observa-se que os veículos que se movem em baixas velocidades (15 e $30 \mathrm{~km} / \mathrm{h}$ ) alcançaram um percentual de vazão de $96.18 \%$ e $93.23 \%$ acima de $1 \mathrm{Gbps}$, respectivamente, enquanto veículos em alta velocidade $(60 \mathrm{~km} / \mathrm{h})$ tiveram o percentual de 91.44\%. A Figura 4(b) mostra que os veículos que se movem em alta velocidade (70 $\mathrm{km} / \mathrm{h}$ ) alcançaram um percentual de $92,07 \%$ acima de $1 \mathrm{Gbps}$, enquanto nas velocidades muito altas (140 e $300 \mathrm{~km} / \mathrm{h}$ ), esse percentual é de 90,13\% e 85,94\%, respectivamente.

Por fim, a combinação de SDN com células virtuais proporcionou uma redução na sinalização da rede, uma vez que nenhuma interface X2 é necessária para handovers intra-V-Cell (permitindo que uma zona sem handover possa ser obtida dentro de uma célula virtual). $\mathrm{O}$ controle de congestionamento e balanceamento adotado garantiu e contribuiu na distribuição da carga da rede. Com as decisões centralizadas no controlador, as mensagens são reduzidas pelo fato de que o controlador SDN é o responsável pelas decisões sobre as $\mathrm{V}$-Cells de origem e destino, tornando a solução desenvolvida mais eficaz que as demais propostas. Além disso, o número de handovers frequentes, causados principalmente pela densificação da rede e alta mobilidade veicular, foi reduzido e contribuiu para os resultados alcançados. Todas as operações tiveram um tempo de processamento inferior a $15 \mathrm{~ms}$, que é a referência para uma rede 5G [Prados-Garzon et al. 2016].

\section{Conclusões e Trabalhos Futuros}

Este artigo apresentou uma solução para tomada de decisão de handover para veículos conectados em redes 5G ultradensas. Para minimizar problemas em processos de handover, causados pela alta mobilidade veicular, este trabalho seleciona as melhores células para comporem uma célula virtual. Estas $V$-Cells são atualizadas dinamicamente, o que proporciona handovers mais rápidos e eficazes. A solução seleciona células por qualidade (especificada pelo 3GPP) e utiliza métricas de redes complexas. Os multicritérios utilizados diferenciam este trabalho das soluções propostas na literatura e proporcionam ganhos de desempenho como: diminuição do tempo de handover, diminuição do número de handovers desnecessários (efeito ping-pong) e falhos, e 
diminuição de pacotes perdidos. Desta forma, conclui-se que o trabalho apresentou avanços em relação às métricas avaliadas quando comparadas com os trabalhos da literatura. Com o objetivo de tratar a alta mobilidade veicular e proporcionar ganhos de desempenho em handover para veículos, o controlador SDN permite que um conjunto de informações seja processado com mais eficiência, com visão unificada da rede. Como trabalhos futuros, estão previstas análises de outras técnicas, incluindo abordagens heurísticas, simulação de diferentes cenários e integração de novos elementos.

\section{Agradecimentos}

O presente trabalho foi realizado com apoio da Coordenação de Aperfeiçoamento de Pessoal de Nível Superior (CAPES) - Código de Financiamento 001. Os autores agradecem também ao Conselho Nacional de Desenvolvimento Científico e Tecnológico (CNPq) e à Fundação de Amparo à Pesquisa do Estado de Minas Gerais (Fapemig). Este trabalho foi apoiado em parte também pelo CEFET-MG, pelo IFMG e pela PUC Minas.

\section{Referências}

Bilen, T., Canberk, B., and Chowdhury, K. R. (2017). Handover Management in Software-Defined Ultra-Dense 5G Networks. IEEE Network, 31(4):49-55.

Gharsallah, A., Zarai, F., and Neji, M. (2018). SDN/NFV-based handover management approach for ultradense 5G mobile networks. Int. Journal of Communication Systems, 0(0):e3831.

ITU (2015). Recommendation ITU-R M.2083-0: IMT Vision - Framework and Overall Objectives of the Future Development of IMT for 2020 and Beyond.

Lousada, E., Storck, C., Mini, R., and Duarte-Figueiredo, F. (2019). Protocolo Baseado em Métricas de Redes Complexas para Mitigação de Tempestade de Broadcast. In XI Simpósio Brasileiro de Computação Ubíqua e Pervasiva, SBCUP 2019.

Minoli, D. and Occhiogrosso, B. (2019). Practical Aspects for the Integration of 5G Networks and IoT Applications in Smart Cities Environments. Wireless Comm. and Mob. Computing, 2019:1-30.

Mouawad, N., Naja, R., and Tohme, S. (2018). User-Centric vs Network-Centric Vertical Handover Algorithms in 5G Vehicular Networks. In Int. Conf. on Internet of Vehicles, pages 46-59.

Nguyen, T. V., Shailesh, P., Sudhir, B., Kapil, G., Jiang, L., Wu, Z., Malladi, D., and Li, J. (2017). A Comparison of Cellular Vehicle-to-Everything and Dedicated Short Range Communication. In 2017 IEEE Vehicular Networking Conference (VNC), pages $101-108$

Oliveira, G., Storck, C. R., and Duarte-Figueiredo, F. (2019). SoftH: Soft Handover Multicriteria Mechanism. In 34th ACM/SIGAPP Symposium On Applied Computing, pages 00884-00889.

Peng, H., Shen, X., and Li, G. Y. (2019). Vehicular Communications: A Network Layer Perspective. IEEE Transactions on Vehicular Technology, 68(2):1064-1078.

Polese, M., Giordani, M., Mezzavilla, M., Rangan, S., and Zorzi, M. (2017). Improved Handover Through Dual Connectivity in 5G mmwave Mobile Networks. Sel. Areas in Communications, 35(9):2069-2084.

Prados-Garzon, J., Adamuz-Hinojosa, O., Ameigeiras, P., Ramos-Munoz, J. J., Andres-Maldonado, P., and Lopez-Soler, J. M. (2016). Handover implementation in a 5G SDN-based mobile network architecture. In Int. Symposium on Personal, Indoor, and Mobile Radio Communications, pages 1-6.

Rizvi, H. and Akram, J. (2018). Handover Management in 5G Software Defined Network Based V2X Communication. In Int. Conference on Open Source Systems and Technologies, pages 22-26.

Silva, F. A., Boukerche, A., Silva, T. R. M. B., Cerqueira, E., Ruiz, L. B., and Loureiro, A. A. F. (2019). Information-Driven SoftwareDefined Vehicular Networks: Adapting Flexible Architecture to Various Scenarios. IEEE Vehicular Technology Magazine, 14(1):98-107.

Souza, D. D. S., Vieira, R. F., Seruffo, M. C. D. R., and Cardoso, D. L. (2020). A Novel Heuristic for Handover Priority in Mobile Heterogeneous Networks. IEEE Access, 8:4043-4050.

Tayyab, M., Gelabert, X., and Jäntti, R. (2019). A Survey on Handover Management: From LTE to NR. IEEE Access, 7:118907118930.

Vukadinovic, V., Bakowski, K., Marsch, P., Garcia, I. D., Xu, H., Sybis, M., Sroka, P., Wesolowski, K., Lister, D., and Thibault, I. (2018). 3GPP C-V2X and IEEE 802.11p for Vehicle-to-Vehicle communications in highway platooning scenarios. Ad Hoc Networks, 74:17-29.

Zang, S., Bao, W., Yeoh, P. L., Vucetic, B., and Li, Y. (2019). Managing Vertical Handovers in Millimeter Wave Heterogeneous Networks. IEEE Transactions on Communications, 67(2):1629-1644. 